\title{
P02-305
}

\section{MEDICATING MALINGERERS: A CASE STUDY OF FEIGNED SICKLE CELL DISEASE}

A. Welch ${ }^{1}$, S. Sachdeva ${ }^{1}$, C. Chung ${ }^{2}$, A. Alao ${ }^{1}$

${ }^{1}$ Department of Psychiatry- Consultation Liaison, ${ }^{2}$ SUNY Upstate Medical University, Syracuse, USA

Speculation exists that bone pain crises among sickle cell disease (SCD) patients are not adequately treated. We counter this assertion with the case of a 17 year-old African American woman receiving narcotics while malingering SCD.

There are various complications of SCD with the most common resulting from ischemia of the bone marrow. While depression, anxiety, and post-traumatic stress disorder have been described, psychiatric complications are not well documented in SCD.

A 17 year old African American female entered the emergency room (ER) with right tibia and fibula fractures. She admitted to having SCD with bone pain crises previously treated with Ketorolac and Meperidine.

On this occasion, radiological studies did not confirm sickle cell changes. A subsequent immunoglobulin electrophoresis came back hemoglobin AA. After the patient received notification she did not have SCD, she attempted to leave against medical advice. Her fractures were treated and she was discharged.

DSM IV defines malingering as "the intentional production of false or grossly exaggerated physical or psychological symptoms". One previous report of malingering sickle cell crises exists. ${ }^{1}$ This case demonstrates that the motivation to gain narcotic analgesics is a determining factor for malingering vis-à-vis factitious disorder, in which the primary goal is to assume the sick role. ${ }^{1}$

Clinicians may assume the validity of a self-reported SCD history. In this case, ER physicians prescribed narcotics without objective evidence of SCD pathology. We suggest verification of SCD diagnosis in order to prevent unnecessary prescription of narcotics.

1. Chang, J.T., Szczyglinski, J.A., King, S.A. A case of malingering: Feigning a painful disorder in the presence of true medical illness. Pain Medicine 2000;1: 280-281. 